\title{
Roseomonas mucosa
}

National Cancer Institute

\section{Source}

National Cancer Institute. Roseomonas mucosa. NCI Thesaurus. Code C124372.

A species of Gram-negative cocco bacillus in the family Acetobacteraceae. R. mucosa is characterized by the formation of mucoid, runny colonies. The species has urease activity and assimilates arabinose, maltose, citrate, and glucose. 